\title{
Optical properties of chitosan/hydroxyl-functionalized graphene quantum dots thin film for potential optical detection of ferric (III) ion
}

\begin{abstract}
In this research, the preparation of chitosan and hydroxyl-functionalized graphene quantum dots (HGQDs) composite has been described. The spin coating technique was used to prepare the chitosan/hydroxyl-functionalized graphene quantum dots (Cs/HGQDs) thin film. The Cs/HGQDs thin film was then characterized using the Fourier transform infrared spectroscopy which confirmed the existence of amino groups, carboxylic acid groups, carboxyl groups and hydroxyl groups in Cs/HGQDs. UV-Vis absorption spectroscopy and photoluminescence (PL) were used to study the optical properties of the thin film. The absorption of Cs/HGQDs thin film was high with optical band gap of $3.797 \mathrm{eV}$. The intensity of PL spectra of the thin film was observed around wavelength of $420 \mathrm{~nm}$. The incorporation of Cs/HGQDs thin film with surface plasmon resonance spectroscopy produced positive responses towards the $\mathrm{Fe}^{3+}$ ion solutions of different concentration and it was found that Cs/HGQDs thin film able to detect $\mathrm{Fe}^{3+}$ as low as $0.5 \mathrm{ppm}$ with a sensitivity of $0.11396^{\circ} \mathrm{ppm}^{-1}$. Subsequently, Cs/HGQDs layer have high potential as sensing layer to detect $\mathrm{Fe}^{3+}$ due to high affinity of $\mathrm{Fe}^{3+}$ ion towards the thin film with a value of binding affinity constant, $\mathrm{K}$ equals to $5.79 \mathrm{ppm}^{-1}$. Atomic force microscopy was used to observe the surface morphology of the thin film and the result indicates that the thin film is relatively smooth and homogenous which also confirmed the interaction of $\mathrm{Fe}^{3+}$ with the thin film. Thus, Cs/HGQDs thin film shows potential for the detection of $\mathrm{Fe}^{3+}$ in solution.
\end{abstract}

Keyword: Surface plasmon resonance; Optical properties; Graphene; Quantum dots; Sensing; Ferric ion 